\title{
AUXILIARY PROBLEM PRINCIPLE EXTENDED TO MONOTONE VARIATIONAL INEQUALITIES
}

\author{
AKHTAR A. KHAN
}

\begin{abstract}
In the present work, the use of the operator method of regularization in the sense of Tikhonov, which makes it possible to develop an iterative scheme via auxiliary problem principle, converging strongly towards the solution of multivalued monotone variational inequality within the absence of strong monotonicity condition of involved operator, is sustained.
\end{abstract}

\section{Introduction}

In recent years, variational inequality theory has emerged as a powerful and effective technique to study a wide range of unrelated free, moving and equilibrium problems in various branches of pure and applied sciences in a unified and general framework. There is already in the literature a substantial number of iterative algorithms including the projection methods, for obtaining the numerical solution of variational inequalities, (cf. e.g. $[3,10,13])$. It is uniformly accepted that the scope of projection algorithms is limited, due to the fact that it is very difficult to find the projection of the space into underlying convex set except in very simple cases. Also a number of variational inequality formulations exist where projection methods are not effective, (cf. e.g. $[1,8,10]$ ). Motivated by these strong reasons a number of workers proposed alternative techniques. One notable step was taken in this direction by Cohen [6] when he succeeded to extend the so called auxiliary problem principle having its origin in optimization problems to solve variational inequalities. While developing an iterative scheme via auxiliary problem principle one imposes several conditions on the data, particularly it is required that the involved operator should be strongly monotonic. Unfortunately this assumptions exclude some of the most important applications such as some typical problems of convex programming and mathematical economics, (cf.e.g. $[2,7,10]$ ). However in [6] author has raised the

Received October 6, 1994.

1991 Mathematics Subject Classification. 49J40, 650K.

Key words and phrases. Variational inequalities, auxiliary principle, Tikhonov's regularization, complementarity problems. 
question whether it is possible to maintain the strong convergence of iterative scheme developed via auxiliary problem principle to the solution of variational inequality within the absence of strong monotonicity of involved operator. In the present work we have succeeded to provide a positive answer to the aforementioned question by utilizing the well known Tikhonov regularization recently considered by Isac [11] in the context of nonlinear comlementarity problems, see also $[2,3,5]$.

Rest of the paper is organised in three sections. $\S 2$, contains some preliminaries to be used throughout the paper. In $\S 3$, we introduce the regularized variational inequality and establish its strong convergence to the minimum norm solution of the original problem. $\S 4$ is devoted to the unification of auxiliary problem principle and Tikhonov regularization by the introduction of regularized auxiliary problem which is shown stable.

\section{Preliminaries}

All of our results are set in a reflexive Banach space $X$ with its dual $X^{*}$. Let $F: D(F) \rightarrow X^{*}$ where $D(F) \subset X$, be a maximal monotone point to set operator, $K$ be a nonempty closed convex subset of $X, \phi$ a lower semicontinuous convex functional from $X$ to $(-\infty,+\infty]$. Let $f$ be an arbitrary element of $X$.

Let $x_{1}, x_{2} \in D(F), y_{1} \in F x_{1}, y_{2} \in F x_{2}$. Recall that $F$ is called monotone, if

$$
<y_{1}-y_{2}, x_{1}-x_{2}>\geq 0
$$

strictly monotone, if

$$
<y_{1}-y_{2}, x_{1}-x_{2}>=0 \text { only if } x_{1}=x_{2} ;
$$

strongly monotone, if

$$
<y_{1}-y_{2}, x_{1}-x_{2}>\geq c\left\|x_{1}-x_{2}\right\|^{2} ; c>0,
$$

maximal monotone, if the graph of $F$ is not properly contained in the graph of any other monotone operator with same domain,

Lipschitz continuous, if

$$
\left\|y_{1}-y_{2}\right\| \leq L\left\|x_{1}-x_{2}\right\|, L>0
$$

We shall investigate the following variational inequality (V.I.) problem: Find $x \in K$ such that for some $y \in F x$ and all $z \in K$

$$
<y, z-x>+\phi(z)-\phi(x) \geq<f, z-x>
$$

An element satisfying above inequality will be called a solution of (2.5). Let us denote the set of all such solutions $x$ by $S$. 
Following two results are now classical and can be obtained from [3,4,12].

Lemma 2.1. Let $X$ be a reflexive Banach space with its dual $X^{*}$ and let $F: D(F) \rightarrow X^{*}$, where $D(F) \subseteq X$ be a maximal monotone point to set mapping. Let $K$ be a nonempty closed convex subset of $X$ satisfying int $K \neq \phi, K \subseteq D(F)$, $\phi$ a lower semicontinuous convex function from $X$ to $(-\infty,+\infty)$ with $\phi \not \equiv \infty$. Then an element $x \in K$ will be a solution of (2.5) if and only if it is a solution of equivalent variational inequality

$$
<u, z-x>+\phi(z)-\phi(x) \geq<f, z-x>, u \in F z, \text { for all } z \in K \text {. }
$$

Lemma 2.2. If abongwith the hypothesis of Lemma 2.1 the following coercive condition

$$
\operatorname{Lim}[<y, x>+\phi(x)] /\|x\| \rightarrow \infty \text { as }\|x\| \rightarrow \infty \quad \text { for } y \in F x, x \in K
$$

be satisfied. Then the set of solutions of (2.5) is nonempty. Further, if the operator $F$ is strictly monotone, solution is unique.

Remark 2.1. It is obvious that the solution set $S$ (possible empty) is closed and convex.

\section{Regularization of Variatonal Inequalities}

Let $\left\langle\epsilon_{n}\right\rangle$ for $n \in N$ be a sequence of positive reals which is strictly decreasing and converging towards zero.

For every $n \in N$ we denote $F_{n} \equiv F+\epsilon_{n} I$, and we say that $F_{n}$ is the Tikhonov regularization of order $\epsilon_{n}$ of operator $F$. Here $I$ is the identity operator.

We shall now consider the regularized variatoinal inequality (R.V.I.) problem: Find $x_{\epsilon_{n}} \in K$ such that for some $y_{\epsilon_{n}} \in F x_{\epsilon_{n}}$ and all $z \in K$

$$
<y_{\epsilon_{n}}+\epsilon_{n} x_{\epsilon_{n}}, z-x_{\epsilon_{n}}>+\phi(z)-\phi\left(x_{\epsilon_{n}}\right) \geq<f, z-x_{\epsilon_{n}}>
$$

Our first result, an existence theorem for (3.1) is as follows.

Theorem 3.1. Let $X$ be a reflexive Banach space with its dual $X^{*}$ and let $F: D(F) \rightarrow X^{*}$ where $D(F) \subseteq X$ be a maximal monotone point to set mapping. Let $K$ be a nonempty closed convex subset of $X$ satisfying int $K \neq \phi, K \subseteq D(F)$, $\phi$ a lower semicontinuous convex function from $X$ to $(-\infty,+\infty]$ with $\phi \not \equiv+\infty$. Let $f$ be an arbitrary element of $X$. Then for each $\epsilon_{n}$ there exists a unique regularized solution $x_{\epsilon_{n}}$ of (3.1). 
Proof. Since the operator $F$ is monotonic, for $y \in F x, y_{1} \in F z$, we have

$$
<y, x-z>\geq-\left\|y_{1}\right\|\|x-z\| \text {. }
$$

Also, since $\phi$ is a proper convex lower semicontinuous functional, for every $z \in$ int $\operatorname{dom}(\phi)$, $\partial \phi(z) \neq \phi$, we have

$$
\begin{aligned}
\phi(x) & \geq \phi(z)+<r, x-z>; \forall r \in \partial \phi(z), \text { for all } x \\
\Rightarrow \phi(x) & \geq \phi(z)-\|r\|\|x-z\| ; \forall r \in \partial \phi(z), \text { for all } x .
\end{aligned}
$$

Now from (3.2) and (3.3), we obtain

$$
\left\{\left\langle y+\epsilon_{n} x, x-z>+\phi(x)\right\} \geq \phi(z)+\left\{-\epsilon_{n}\|x\|-\|r\|-\left\|y_{1}\right\|\right\}\|x-z\|\right.
$$

which implies the coercivity of $\left\{F_{n}(x)+\phi(x)\right\}$ on $K$. Thus by Lemma 2.2 there exists a unique solution $x_{\epsilon_{n}}$ for (3.1).

Theorem 3.2. Under the hypothesis of Theorem 3.1, the set of solutions of (2.5) is nonempty if and only if $\left\langle x_{\epsilon_{n}}\right\rangle$ is bounded.

Proof. Let $S \neq \phi$, that is, there exists $x \in S$. Setting $z=x_{\epsilon_{n}}$ in (2.5) and $z=x$ in (3.1) and adding we obtain

$$
\begin{array}{ll} 
& -<y_{\epsilon_{n}}-y, x_{\epsilon_{n}}-x>+\epsilon_{n}<x_{\epsilon_{n}}, x-x_{\epsilon_{n}}>\geq 0 \\
\Rightarrow \quad & <x_{\epsilon_{n}}, x-x_{\epsilon_{n}}>\geq 0 \\
\Rightarrow \quad\left\|x_{\epsilon_{n}}\right\| \leq\|x\|,
\end{array}
$$

for some $x \in S$. Thus the sequence $\left\langle x_{\epsilon_{n}}\right\rangle$ is bounded.

For the converse, let the sequence $\left\langle x_{\epsilon_{n}}\right\rangle$ is bounded. Since the space $X$ is reflexive, $\left\langle x_{\epsilon_{n}}\right\rangle$ is weakly compact, therefore there must exists a subsequence $\left\langle x_{e_{n}}\right\rangle$ from $\left\langle x_{\epsilon_{n}}\right\rangle$ which converges weakly to $v \in X$. Further, $K$ being closed is weakly closed by Mazur's Lemma, hence $v \in K$. Our aim is now to show that $v \in S$. Using Lemma 2.1, for $u \in F z$, we have

$$
<u+e_{n} z, z-x_{e_{n}}>+\phi(z)-\phi\left(x_{e_{n}}\right) \geq<f, z-x_{e_{n}}>, z \in K
$$

Taking $n \rightarrow \infty, e_{n} \rightarrow 0$ and $x_{e_{n}} \rightarrow v$ weakly, we get

$$
\langle u, z-v>+\phi(z)-\phi(v) \geq\langle f, z-v>, \quad \text { for all } z \in K .
$$

Repeating the use of Lemma 2.1, we get

$$
<w, z-v>+\phi(z)-\phi(v) \geq\langle f, z-v>, w \in F v, z \in K
$$

$$
\Rightarrow v \in S \text {. }
$$


Theorem 3.3. Using the notations above, if the sequence $\left\langle x_{\epsilon_{n}}\right\rangle$ is bounded, $\left\langle x_{\epsilon_{n}}>\rightarrow x^{*}\right.$ as $n \rightarrow \infty$ strongly, where $x^{*}$ is a solution of (2.5) such that

$$
\left\|x^{*}\right\|=\min \{\|x\|: x \in S\}
$$

Proof. Followig the technique of previous theorem, we prove

$$
\begin{aligned}
& <x_{\epsilon_{n}}, x-x_{\epsilon_{n}}>\geq 0 \\
& \Rightarrow<x, x-x_{\epsilon_{n}}>\geq 0 \\
& \Rightarrow<x, x-\bar{x}>\geq 0
\end{aligned}
$$

where $\bar{x}$ is the weak limit of $\left\langle x_{e_{n}}\right\rangle$. First we shall specify $\bar{x}$ as $x^{*}$. For, we set $x_{t}=t \bar{x}+(1-t) x \in S, t \in[0,1]$ for arbitrary $x \in S$ in (3.7), and using the fact $(1-t) \geq 0$, we obtain

$$
<x_{t}, x-\bar{x}>\geq 0
$$

Passing this to limit $t \rightarrow 1$, we have

$$
<\bar{x}, x-\bar{x}>\geq 0
$$

which is (3.5). Since $x^{*}$ is unique, the entire family $\left\langle x_{\epsilon_{n}}\right\rangle$ must be weakly cnvergent to $x^{*}$.

Further, from (3.6), we have

$$
\left\|x_{\epsilon_{n}}-x^{*}\right\|^{2} \leq<x^{*}, x^{*}-x_{\epsilon_{n}}>
$$

In view of the weak convergence of $x_{\epsilon_{n}}$ to $x^{*}$, and (3.8), the theorem now follows.

\section{Regularization Via Auxiliary Problem Principle}

Let us introduce an auxiliary functional $T: K \rightarrow \mathbb{R}$ that we choose convex and Gateaux differentiable, and a positive number $\beta$. For some $x \in K$, we consider the following regularized auxiliary problem : Find $w(x) \in K$ satisfying

$$
\begin{aligned}
& \quad<T^{\prime}(w(x))+\beta y+\beta \epsilon_{n} x-T^{\prime} x, z-w(x)> \\
& +\beta[\phi(z)-\phi(w(x))] \geq \beta<f, z-x>, \quad \text { for all } z \in K, y \in F x
\end{aligned}
$$

We have the following 
Proposition 4.1. If $x$ is the fixed point of $w(x)$, i.e., if $w(x)=x$, then $w(x)$ is a solution of regularized variational inequality (3.1) with arbitrary but fixed $\epsilon_{n}$, denoted, by $x_{\epsilon_{n}}$.

Proof. The proof is straightforward using (4.1).

Lemma 4.2. Let $J$ be a convex functional defined on a reflexive Banach space. Let the Gateaux derivative $J^{\prime}(x)$ of $J$ at $x$ is strongly monotone with constant $m$. Then

$$
J(x)-J(z) \geq<J^{\prime}(x), x-z>+(m / 2)\|x-y\|^{2} .
$$

Proof. For a proof of this, see [9].

In the following result, for the sake of convenience we choose $X$ to be a real Hilbert space.

Theorem 4.3. If alongwith the hypothesis of Theorem 3.3, $T: X \rightarrow \mathbb{R}$ is a proper convex and differentiable functional and if its derivative $T^{\prime}$ is strongly monotone with modulus $m$ over $K$, then there exists a unique solution $x^{n+1}$ to (4.1), with $x^{n}$ substituted for $x$.

Moreover, if $F$ is Lipschitz continuous with modulus $L$ over $K$ and the conditon

$$
M \epsilon_{n}>\beta\left(\epsilon_{n}^{2}+L^{2}\right) \forall \epsilon_{n}>0 .
$$

holds, then we have

$$
\left\|x^{n+1}-x^{*}\right\| \rightarrow 0 \text { as } n \rightarrow \infty
$$

where $x^{*}$ is the minimum norm solution of (2.5).

Proof. First part of the theorem can be easily proved by following the same line as of Theorem 3.1. Here without any harm to generality, we shall assume that $f=0$, see [4] at this point. By the result of Theorem 3.3, we have $\operatorname{Lim}_{n \rightarrow \infty}\left\|x_{\epsilon_{n}}-x^{*}\right\|=0$ where $x^{*}$ is the minimum norm solution of (2.5). Now using this and obvious triangle inequality

$$
\left\|x^{n}-x^{*}\right\| \leq\left\|x^{n}-x_{\epsilon_{n}}\right\|+\left\|x_{\epsilon_{n}}-x^{*}\right\|
$$

it is sufficient to show that (see $[2,3])$

$$
\operatorname{Lim}_{n \rightarrow \infty}\left\|x_{n}-x_{\epsilon_{n}}\right\|=0
$$

For, let us introduce the function

$$
\Phi(x)=T\left(x_{\epsilon_{n}}\right)-T(x)-\left\langle T^{\prime}(x), x_{\epsilon_{n}}-x>.\right.
$$


We have

$$
\begin{aligned}
\Phi\left(x^{n}\right)-\Phi\left(x^{n+1}\right)= & \left\{T\left(x_{\epsilon_{n}}\right)-T\left(x^{n}\right)-<T^{\prime}\left(x^{n}\right), x_{\epsilon_{n}}-x^{n}>\right\} \\
& -\left\{T\left(x_{\epsilon_{n}}\right)-T\left(x^{n+1}\right)-<T^{\prime}\left(x^{n+1}\right), x_{\epsilon_{n}}-x^{n+1}>\right\} \\
= & T\left(x^{n+1}\right)-T\left(x^{n}\right)-<T^{\prime}\left(x^{n}\right), x^{n+1}-x^{n}> \\
& +<T^{\prime}\left(x^{n+1}\right)-T^{\prime}\left(x^{n}\right), x_{\epsilon_{n}}-x^{n+1}> \\
\geq & (m / 2)\left\|x^{n}-x^{n+1}\right\|^{2} \\
& +<T^{\prime}\left(x^{n+1}\right)-T^{\prime}\left(x^{n}\right), x_{\epsilon_{n}}-x^{n+1}>
\end{aligned}
$$

by using (4.2).

Now, by substituting $x=x^{n}, w(x)=x^{n+1}$ and $z=x_{\epsilon_{n}}$ in (4.1) we obtain

$<T^{\prime}\left(x^{n+1}\right)+\beta y^{n}+\beta \epsilon_{n} x^{n}-T^{\prime}\left(x^{n}\right), x_{\epsilon_{n}}-x^{n+1}>+\beta\left[\phi\left(x_{\epsilon_{n}}\right)-\phi\left(x^{n+1}\right)\right] \geq 0, y^{n} \in F x^{n}$.

$\Rightarrow<T^{\prime}\left(x^{n+1}\right)-T^{\prime}\left(x^{n}\right), x_{\epsilon_{n}}-x^{n+1}>$

$\geq-\beta\left[<y^{n}+\epsilon_{n} x^{n}, x_{\epsilon_{n}}-x^{n+1}>+\phi\left(x_{\epsilon_{n}}\right)-\phi\left(x^{n+1}\right)\right]$,

On the other hand if we substitute $x^{n+1}$ for $z$ in (3.1) we obtain

$$
\phi\left(x^{n+1}\right)-\phi\left(x_{\epsilon_{n}}\right) \geq-<y_{\epsilon_{n}}+\epsilon_{n} x_{\epsilon_{n}}, x^{n+1}-x_{\epsilon_{n}}>, y_{\epsilon_{n}} \in F x_{\epsilon_{n}} .
$$

Combining (4.3), (4.4) and (4.5) we obtain

$$
\begin{aligned}
\Phi\left(x^{n}\right)-\Phi\left(x^{n+1}\right) \geq & (m / 2)\left\|x^{n}-x^{n+1}\right\|^{2}+\beta<y^{n}+\epsilon_{n} x^{n}, x^{n+1}-x_{\epsilon_{n}}> \\
& -\beta<y_{\epsilon_{n}}+\epsilon_{n} x_{\epsilon_{n}}, x^{n+1}-x_{\epsilon_{n}}> \\
\geq & (m / 2)\left\|x^{n}-x^{n+1}\right\|+\beta<y^{n}-y_{\epsilon_{n}}+\epsilon_{n}\left(x^{n}-x_{\epsilon_{n}}\right), x^{n+1}-x_{\epsilon_{n}}> \\
\geq & (m / 2)\left\|x^{n}-x^{n+1}\right\|^{2}+\beta<y^{n}-y_{\epsilon_{n}}+\epsilon_{n}\left(x^{n}-x \epsilon_{n}\right), x^{n+1}-x^{n}> \\
& +\beta<y^{n}-y_{\epsilon_{n}}+\epsilon_{n}\left(x^{n}-x_{\epsilon_{n}}\right), x^{n}-x_{\epsilon_{n}}> \\
\geq & (m / 2)\left\|x^{n}-x^{n+1}\right\|^{2}+\beta<y^{n}-y_{\epsilon_{n}}, x^{n+1}-x^{n}> \\
& +\beta \epsilon_{n}<x^{n}-x_{\epsilon_{n}}, x^{n+1}-x^{n}>+\beta<y^{n}-y_{\epsilon_{n}}, x^{n}-x_{\epsilon_{n}}> \\
& +\beta \epsilon_{n}<x^{n}-x_{\epsilon_{n}}, x^{n}-x_{\epsilon_{n}}> \\
\geq & (m / 2)\left\|x^{n}-x^{n+1}\right\|^{2}+\beta \epsilon_{n}\left\|x^{n}-x_{\epsilon_{n}}\right\|^{2} \\
& -\beta\left\|y^{n}-y_{\epsilon_{n}}\right\|\left\|x^{n+1}-x^{n}\right\|-\beta \epsilon_{n}\left\|x^{n}-x \epsilon_{n}\right\|\left\|x^{n+1}-x^{n}\right\| \\
\geq & (m / 2)\left\|x^{n}-x^{n+1}\right\|^{2}+\beta \epsilon_{n}\left\|x^{n}-x_{\epsilon_{n}}\right\|^{2} \\
& -\left(\beta^{2} / m\right)\left\|y^{n}-y_{\epsilon_{n}}\right\|^{2}-(m / 4)\left\|x^{n+1}-x^{n}\right\|^{2} \\
& -\left(\beta^{2} \epsilon_{n}^{2} / m\right)\left\|x^{n}-x_{\epsilon_{n}}\right\|^{2}-(m / 4)\left\|x^{n+1}-x^{n}\right\|^{2} \\
\geq & \beta \epsilon_{n}\left\|x^{n}-x_{\epsilon_{n}}\right\|^{2}-\left(L^{2} \beta^{2} / m\right)\left\|x^{n}-x_{\epsilon_{n}}\right\|^{2} \\
& -\left(\beta^{2} \epsilon_{n}^{2} / m\right)\left\|x^{n}-x_{\epsilon_{n}}\right\|^{2} \\
= & {\left[\beta \epsilon_{n}-\left(\beta^{2} \epsilon_{n}^{2}+L^{2} \beta^{2}\right) / m\right]\left\|x^{n}-x \epsilon_{n}\right\|^{2} } \\
= & (\beta / m)\left[m \epsilon_{n}-\beta\left(\epsilon_{n}^{2}+L^{2}\right)\right]\left\|x^{n}-x_{\epsilon_{n}}\right\|^{2} \\
&
\end{aligned}
$$


Now by the hypothesis (A), (4.6) confirms that the nonnegative sequence $\Phi\left(x^{n}\right)$ is strictly decreasing and by the classical arguments we lead to the conclusion that $x^{n}$ strongly converges towards $x_{\epsilon_{n}}$, i.e., $\left\|x^{n}-x_{\epsilon_{n}}\right\| \rightarrow 0$ as $n \rightarrow \infty$. This establishes the theorem.

\section{Concluding Remarks}

In the present work, we have extended the so called auxiliary problem principle [cf.e.g.[6]) to solve multivalued monotone variational inequalities without imposing any condition of strong monotonicity on the involved operator by utilizing the Tikhonov's regularization (cf.e.g.[11,15]. The idea of such unification was previously considered by Bakushinski in [3]. Our results are quite flexible and one may apply similar techniques to solve nonlinear monotone complementarity problems (cf.e.g. [14]) in the absence of strong monotonicity condition or saddle point problem (cf.e.g. [6]) without assuming the strong convexity-concavity of the involved function.

\section{References}

[1] C. Baiocchi and A. Capelo, "Variational and quasi-variational inequalities, applications to free boundary value problems", Wiley, New York, 1984.

[2] A. B. Bakushinsky, "Methods for solving monotone variational inequalities based on the iterative regularization principle," USSR Comp. Maths. Math. Phy., 17(1977), 1350-1362.

[3] A. B. Bakshinsky and B. T. Poljak, "On the solution of variational inequalities," Soviet Math. Dokl., 15(1974), 1705-1710.

[4] F. E. Browder, "On the unificaiton of the calculus of variations and the theory of monotone nonlinear operators in Banach spaces," Proc. Nat. Acad. Sci. USA, 56(1966), 419-425.

[5] F. E. Browder, "Existence and approximation of solution of nonlinear variational inequalities," 56(1966), 1080-1086.

[6] G. Cohen, Auxiliary problem principle extended to variational inequalities,JOTA, 59(1988), 325333.

[7] S. Dafermos, "Traffic equilibria and variational inequalities," Trans. Sci., 14(1980), 42-54.

[8] G. Duvaut and J. L. Lions, "Les inequations in mechanique et en physique,"Dunod-Paris, 1972 (Eng. Tran., Springer, Berlin, 1976).

[9] I. Ekeland and R. Temam "Analyse convex et problems variationallles," Dunod-Paris, 1974 (Eng. Tran., North Holland, Amsterdam, 1970).

[10] R. Glowinski J. L. Lions and R, Tremolires "Analyse numerique des inequations variationnelles (Tome 1 et 2), Dunod," Paris(Eng. Tran., North Holland, Amsterdam, 1981).

[11] G. Isac "Tikhonov's regularization and the complementarity problem in Hilbert spaces," J. Math. Anal. Appl., 174(1993), 53-66.

[12] L. D. Muu, "An augumented penalty function method for solving a class of variational inequalities," USSR Comp. Maths. Math. Phy., 26(1986), 117-122.

[13] M. A. Noor, "On nonlinear variational inequalities," Inter. J.Math. ES Math. Sci., 14(1991), 399402.

[14] R. Saigal, "Extension of the generalized complementarity problem," Math. Oper. Res., 1(1976), 260-266.

[15] A. N. Tikhonov and V. Y. Arsenin, "Solution of ill posed problems," J.Wiley sons, New York, 1977.

Department of Mathematics, Faculty of Natural Sciences, Jamia Millia Islamia New Delhi-110025, INDIA. 